\title{
Distribuição da matéria seca e composição química das raízes, caule e folhas de goiabeira submetida a estresse salino ${ }^{(1)}$
}

\author{
Raimundo Gonçalves Ferreira ${ }^{(2)}$, Francisco José Alves Fernandes Távora( ${ }^{(2)}$ e Fernando Felipe Ferreyra Hernandez ${ }^{(3)}$
}

Resumo - Um experimento em casa de vegetação foi conduzido com o objetivo de estudar a resposta de plantas de goiabeira (Psidium guajava L.) a diferentes concentrações de $\mathrm{NaCl}$. As plantas foram cultivadas em solução nutritiva, com 0, 25, 50, 75, 100, 125 e $150 \mathrm{mM}$ de $\mathrm{NaCl}$, e colhidas com 30 e 50 dias após estresse, num delineamento inteiramente casualizado. Foram determinadas: matéria seca das raízes e parte aérea; e porcentagens de $\mathrm{Na}, \mathrm{Cl}, \mathrm{K}, \mathrm{Ca}$ e $\mathrm{Mg}$ nas raízes, caule e folhas. O estresse salino reduziu a matéria seca total, e a relação parte aérea/raiz foi reduzida. O estresse causou um aumento nos níveis dos íons $\mathrm{Na}^{+} \mathrm{e} \mathrm{Cl}$, com maior acúmulo nas folhas, seguido das raízes. Os teores de $\mathrm{Ca}$ foram estáveis nas raízes, mas decresceram no caule e folhas. Os teores de $\mathrm{K}$ foram reduzidos, particularmente nas folhas. Ao contrário, os teores de $\mathrm{Mg}$ não foram afetados pela salinidade no caule e nas raízes, havendo, entretanto, redução nas folhas. $\mathrm{O}$ teor de $\mathrm{Na}$ apresentou relação direta com o de $\mathrm{Cl}$ e inversa com o de $\mathrm{K}$ nas raízes e folhas. $\mathrm{O}$ teor de Ca não variou com o de Na nas folhas, mas apresentou uma relação inversa nas raízes. O teor de $\mathrm{Mg}$ não variou com o de Na nas raízes e folhas.

Termos para indexação: Psidium guajava, cloreto de sódio, solução salina, resposta da planta.

Dry matter partitioning and mineral composition of roots, stems and leaves of guava grown under salt stress conditions

\begin{abstract}
A greenhouse experiment was conducted with the objective of studying the response of guava plants (Psidium guajava L.) to increasing levels of $\mathrm{NaCl}$ concentrations. Plants were grown in nutrient solutions, with $0,25,50,75,100,125$ and $150 \mathrm{mM}$ of $\mathrm{NaCl}$, and harvested 30 and 50 days after saline stress in a complete randomized design. The following traits were studied: shoot, root and total plant dry matter and the percentage of $\mathrm{Na}, \mathrm{Cl}, \mathrm{K}, \mathrm{Ca}$ and $\mathrm{Mg}$ in plant dry matter. Dry matter yield decreased when salinity increased. The root/shoot ratio decreased with the saline treatment. Salinity stress caused an increase in the levels of $\mathrm{Na}^{+}$and $\mathrm{Cl}^{-}$. The highest ion accumulation was found in the leaves followed by the roots. The Ca levels were stable in the roots, but decreased in stems and leaves. The K content was reduced with the increased levels of salinity, particularly in the leaves. On the other hand, $\mathrm{Mg}$ levels were not affected by salinity in stems and roots but decreased in the leaves. There was a positive relationship between $\mathrm{Na}$ and $\mathrm{Cl}$ and a negative relationship between $\mathrm{Na}$ and $\mathrm{K}$ concentration in roots and leaves. $\mathrm{Mg}$ concentration in leaves and roots did not vary with the concentration of Na. The concentration of $\mathrm{Ca}$ did not vary with $\mathrm{Na}$ in the leaves but showed an inverse relationship in the roots.
\end{abstract}

Index terms: Psidium guajava, sodium chloride, salt solutions, plant response.

\section{Introdução}

A ocorrência de solos salinos e sódicos é comum nas regiões áridas e semi-áridas, pela baixa precipi-

\footnotetext{
(1) Aceito para publicação em 4 de fevereiro de 2000.

(2) Universidade Federal do Ceará (UFC), Dep. de Fitotecnia, Caixa Postal 12168, CEP 60455-740 Fortaleza, Ceará E-mail: tavora@ufc.br

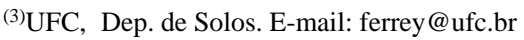

tação pluvial e alta taxa de evaporação. Por isso, os sais não são lixiviados, acumulando-se em quantidades prejudiciais ao crescimento e desenvolvimento das plantas. O Nordeste brasileiro apresenta um grande potencial para o cultivo de plantas frutíferas, entre elas a goiabeira, em áreas irrigadas. Estas áreas apresentam tendência para o acúmulo de sais.

$\mathrm{O} \mathrm{NaCl}$ provoca a redução na absorção e transporte de elementos minerais essenciais ao desenvolvimento e crescimento das plantas. O desequilíbrio 
nutricional constitui um importante componente do estresse salino. A adaptação de glicófitas à salinidade é freqüentemente associada à exclusão de $\mathrm{Na}^{+} \mathrm{e} \mathrm{Cl}$ do tecido foliar (Tsuchiya et al., 1994; Storey, 1995). Comumente são observados, em resposta à aplicação de $\mathrm{NaCl}$, aumento na concentração dos íons $\mathrm{Na}^{+}$ e $\mathrm{Cl}^{-}$, e redução nos níveis de $\mathrm{K}^{+}$e $\mathrm{Ca}^{2-}$ (Shybank \& Kasshirad, 1978; Stolzy \& Jarrel, 1983; Shannon et al., 1998). O aumento na concentração de $\mathrm{Na}^{+}$no ambiente radicular pode causar a redução na absorção de $\mathrm{K}^{+}$. No entanto, a concentração que determina esta redução varia com a espécie, podendo este fato estar associado com sua tolerância à salinidade. Em citrus, o acúmulo do íon $\mathrm{Cl}^{-}$apresenta-se mais prejudicial ao crescimento e produção que o de $\mathrm{Na}^{+}$. Há uma interação entre íons específicos, como por exemplo: a deficiência de $\mathrm{Ca}^{2+}$ pode ser induzida pelo excesso de $\mathrm{Na}^{+}$ou $\mathrm{SO}_{4}{ }^{2-}$, e a de $\mathrm{K}^{+}$por excesso de $\mathrm{Na}^{+}$ou $\mathrm{Ca}^{2+}$, assim como altas concentrações de $\mathrm{Mg}^{2+}$ podem inibir a absorção de $\mathrm{K}^{+}$ou de $\mathrm{Ca}^{2+}$ (Bernstein, 1975). Vários estudos sobre ação dos íons nas plantas revelam que o $\mathrm{Cl}^{-}$é o ânion mais tóxico, e o $\mathrm{Na}^{+}$, o cátion que mais afeta o metabolismo (Stolzy \& Jarrel, 1983).

Os estudos sobre os efeitos do $\mathrm{Na}^{+}$em espécies arbóreas são ainda muito limitados. A literatura registra que a lesão provocada pelo $\mathrm{Na}^{+}$em concentrações inferiores a $5 \mathrm{mmol} / \mathrm{L}$ na solução do solo é comum em abacate (Persea americana Mill), citrus (Citrus spp.) e em frutas de caroço (Prunus spp.). Após a absorção pelas raízes, o $\mathrm{Na}^{+}$é translocado para a parte aérea da planta, causando a queima-dasfolhas dessas espécies (Rhoades \& Loveday, 1990).

A maioria das espécies frutíferas cultivadas é classificada como sensível aos sais (Rhoades \& Loveday, 1990). Há um reduzido número de estudos sobre o efeito da salinidade na produção, crescimento e fisiologia da goiabeira (Desai \& Singh, 1980; Patil et al., 1984). O conhecimento da tolerância da espécie quando cultivada em solo salino é muito importante para que técnicas alternativas de manejo possam ser utilizadas com a finalidade de amenizar os efeitos prejudiciais dos sais.

O objetivo deste trabalho foi estudar os efeitos do estresse salino sobre o crescimento e composição química das raízes, caule e folhas de goiabeira (variedade Rica).

\section{Material e Métodos}

O experimento foi conduzido em casa de vegetação, na Universidade Federal do Ceará, Campus do Pici, em Fortaleza, Ceará, nos meses de setembro a dezembro de 1997. Foram utilizadas mudas de goiabeira (Psidium guajava L.), variedade Rica, produzidas a partir de sementes fornecidas pela Embrapa-Centro Nacional de Pesquisa de Agroindústria Tropical. As plantas foram cultivadas em recipientes de plástico, com $20 \mathrm{~cm}$ de altura, $10 \mathrm{~cm}$ de diâmetro e cinco litros de capacidade, em solução nutritiva de Hoagland \& Arnon (1950), com a seguinte composição química: $\mathrm{K}_{2} \mathrm{SO}_{4} 0,5 \mathrm{M}, 2 \mathrm{~mL} \mathrm{~L}^{-1} ; \mathrm{KH}_{2} \mathrm{PO}_{4} 1 \mathrm{M}$, $1 \mathrm{~mL} \mathrm{~L}^{-1} ; \mathrm{MgSO}_{4} 1 \mathrm{M}, 1 \mathrm{~mL} \mathrm{~L}^{-1} ; \mathrm{Ca}\left(\mathrm{NO}_{3}\right)_{2} 1 \mathrm{M}, 4 \mathrm{~mL} \mathrm{~L}^{-1}$; $\left(\mathrm{NH}_{4}\right)_{2} \mathrm{SO}_{4} 1 \mathrm{M}, 1 \mathrm{~mL} \mathrm{~L}^{-1} ; \mathrm{FeSO}_{4} 1 \mathrm{M}, 4 \mathrm{~mL} \mathrm{~L}^{-1}$; EDTA $0,08 \mathrm{M}, 1 \mathrm{~mL} \mathrm{~L}^{-1}$. Em cada litro de solução nutritiva foi adicionado $1 \mathrm{~mL}$ de solução com micronutrientes contendo $\mathrm{H}_{3} \mathrm{BO}_{3}\left(2,86 \mathrm{~g} \mathrm{~L}^{-1}\right) ; \mathrm{MnCl}_{2} .4 \mathrm{H}_{2} \mathrm{O}\left(1,81 \mathrm{~g} \mathrm{~L}^{-1}\right)$; $\mathrm{ZnSO}_{4} .7 \mathrm{H}_{2} \mathrm{O}\left(0,22 \mathrm{~g} \mathrm{~L}^{-1}\right) ; \mathrm{CuSO}_{4} \cdot \mathrm{H}_{2} \mathrm{O}\left(0,08 \mathrm{~g} \mathrm{~L}^{-1}\right)$, e $\mathrm{H}_{2} \mathrm{MoO}_{4} \cdot \mathrm{H}_{2} \mathrm{O}\left(0,02 \mathrm{~g} \mathrm{~L}^{-1}\right)$.

As sementes foram colocadas para germinar em canteiros contendo areia fina peneirada. O nível de umidade do substrato durante a germinação e desenvolvimento das plantas na sementeira foi mantido próximo à capacidade máxima de retenção de água, por meio de irrigações diárias. Quando as plantas apresentaram quatro pares de folhas definitivas, foram transferidas para os recipientes de plástico contendo solução nutritiva aerada. Foram utilizadas duas plantas por vaso. Em uma primeira fase, com duração de cinco dias, as mudas ficaram em contato direto com a solução nutritiva diluída 1:4, e numa segunda fase, até o décimo dia, a solução foi diluída na razão 1:2, e a partir de então, as plantas ficaram em contato permanente com a solução normal. Depois de 30 dias em solução nutritiva, as plantas foram submetidas ao estresse salino $(\mathrm{NaCl})$, sob diferentes concentrações: 0 (controle), 25, $50,75,100,125$ e $150 \mathrm{mM}$, correspondendo aos seguintes valores de condutividade elétrica (CE): 1,6, 4,5, 7,1, 9,7, 11,3, 13,9 e 16,2 dS m${ }^{-1}$, respectivamente.

Em todas as unidades experimentais o $\mathrm{pH}$ da solução nutritiva foi monitorado diariamente, com o uso de peagômetro de campo, e ajustado próximo de 6,0 , usando-se ácido clorídrico ou hidróxido de sódio 0,1M. Diariamente, também, foi realizada a reposição da água evapotranspirada usando-se água destilada, e semanalmente a solução nutritiva foi trocada.

O cultivo foi mantido 50 dias após o estresse salino (DAES), período em que foram realizadas duas colheitas: aos 30 e aos 50 DAES. Nas colheitas, as plantas foram divididas em raízes, caule e folhas, sendo postas a secar a $70^{\circ} \mathrm{C}$ em estufa com ar forçado, para a determinação da 
matéria seca. Após secagem e pesagem, procedeu-se às análises dos teores de $\mathrm{Na}, \mathrm{Cl}, \mathrm{K}, \mathrm{Ca}$ e $\mathrm{Mg}$. Os teores de $\mathrm{Ca}$ e $\mathrm{Mg}$ foram medidos por espectrofotometria de absorção atômica, e o K e o Na, por fotometria de chama (Miyazawa et al., 1984). O Cl foi extraído por agitação em água destilada, e determinado por titulação com nitrato de prata (Malavolta et al., 1989).

$\mathrm{O}$ experimento constou de um arranjo fatorial ( $7 \times 2)$ de sete concentrações de $\mathrm{NaCl}$ e duas épocas de colheita, num delineamento inteiramente casualizado, com três repetições. Para avaliar os efeitos dos diferentes níveis de salinidade $(\mathrm{NaCl})$ nas diversas variáveis estudadas, foi utilizado o método convencional de análise de variância, mediante a aplicação do teste F. Foram ajustadas funções que relacionaram as variáveis aos níveis de salinidade aplicados.

\section{Resultados e Discussão}

\section{Matéria seca}

A matéria seca da parte aérea aos 30 e 50 DAES foi significativamente afetada pela salinidade. Na Figura 1, constata-se uma relação linear inversa entre níveis de salinidade e matéria seca das raízes e da parte aérea nas duas datas amostradas. A redução da matéria seca das raízes foi menor que a da parte aérea. Embora os resultados revelem a mesma tendência aos 50 DAES, constata-se uma queda mais acentuada tanto das raízes como da parte aérea, em resposta à salinidade. No nível máximo de salinidade $(150 \mathrm{mM} \mathrm{NaCl})$, as reduções na matéria seca foram nas raízes, de $44 \%$ e $55 \%$, e na parte aérea, de $65 \%$ e $79 \%$, respectivamente aos 30 e 50 DAES. As diferenças com relação à capacidade de produção de matéria seca entre raízes e parte aérea, nas duas datas amostradas, tendem a desaparecer com o aumento da salinidade. A redução observada sugere que os íons absorvidos e transportados para a parte aérea possivelmente excederam o limite necessário ao ajustamento osmótico da planta, e desta forma acarretaram efeitos danosos ao crescimento (Flower \& Yeo, 1986).

Segundo Greenway \& Munns (1980) e Ebert (1998), as árvores frutíferas, geralmente sensíveis ao estresse salino, têm o crescimento vegetativo afetado quando submetidas a concentrações inferiores a $20 \mathrm{mM}$ de $\mathrm{NaCl}$. Há, entretanto, registros de diferenças de comportamento entre espécies frutíferas do
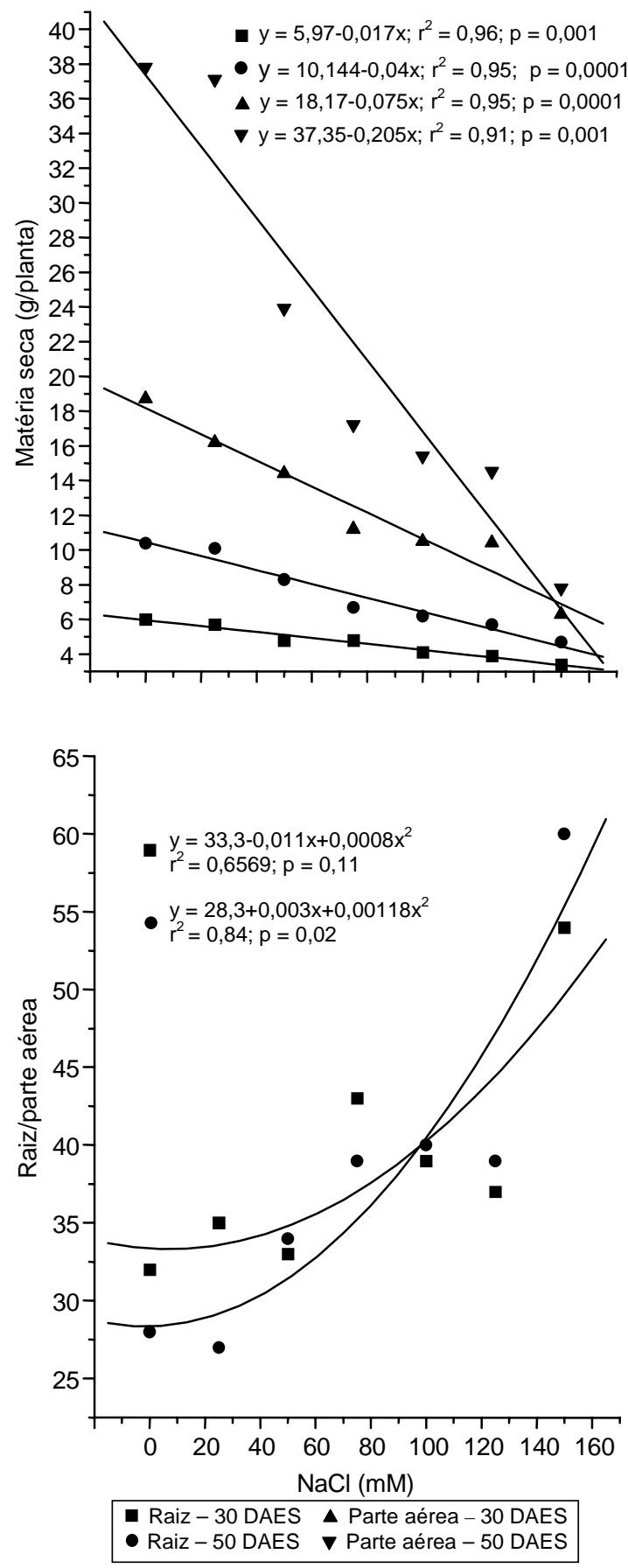

Figura 1. Matéria seca (a) e relação raiz/parte aérea (b) de plantas de goiabeira em razão de níveis de $\mathrm{NaCl}$ na solução nutritiva após 30 e 50 dias de aplicação do estresse salino (DAES). 
gênero Annona. Ebert (1998) não encontrou reduções no crescimento vegetativo em plântulas de A. muricata submetidas a concentração de $60 \mathrm{mM}$ de $\mathrm{NaCl}$. Ao contrário, A. cherimola apresentou redução de $39 \%$ no mesmo nível de salinidade.

Resultados semelhantes foram encontrados por Desai \& Singh (1980), ao estudarem o efeito de diferentes concentrações e tipos de sais em plantas de goiabeira; estes autores também reportaram que o $\mathrm{NaCl}$ foi o sal que mais prejuízos causou ao crescimento das plantas. Patil et al. (1984) constataram, em goiabeira, que todos os parâmetros de crescimento foram afetados pelo sal, principalmente a matéria seca da parte aérea da planta, que sofreu redução de $90 \%$ em relação à do controle, quando foram cultivadas em $60 \mathrm{mM}$ de $\mathrm{NaCl}$.

$\mathrm{Na}$ Figura 1, verifica-se que a relação raiz/parte aérea aumentou com os níveis crescentes de $\mathrm{NaCl}$, seguindo uma equação polinomial do tipo $\mathrm{y}=\mathrm{a}+\mathrm{bx}+\mathrm{cx} \mathrm{x}^{2}$

Constata-se que até níveis próximos de $100 \mathrm{mM}$ de $\mathrm{NaCl}$, esta relação é maior aos 30 DAES, fato que é revertido aos 50 DAES, em que esta relação é maior nos níveis de salinidade superiores a $100 \mathrm{mM}$ de $\mathrm{NaCl}$. Aumentos da relação raiz/parte aérea em resposta à salinidade foram também constatados em algodão (Hoffman et al., 1971), Leucaena leucocephala (Gorham et al., 1988), Suaeda maritima (Yeo \& Flower, 1989), Prosopis juliflora (Jarrel \& Viginia, 1990) e citrus (Storey, 1995). Em contraste, o crescimento da parte aérea de Sorghum bicolor e S. halepense (Yang et al., 1990) e das ornamentais Chrysanthemum sp., Dianthus caryophyllus e Matthiola sp. (Devitt \& Morris, 1987) foi menos inibido pela salinidade do que o da raiz. Tais mudanças morfológicas parecem constituir uma adaptação das plantas ao novo ambiente. $\mathrm{O}$ aumento da relação raiz/parte aérea pode ser atribuído a uma reação do sistema radicular para ampliar a área de exploração de nutrientes e água como forma de amenizar os efeitos prejudiciais do sal. Entretanto, podese especular que a maior redução da parte aérea em relação a raiz pode ser devida à maior sensibilidade aos sais apresentada pelas folhas.

\section{Composição mineral}

O incremento do nível de salinidade na solução nutritiva determinou aumentos significativos nos teores de $\mathrm{Na}$ em todos os órgãos da planta (Tabela 1).
Tabela 1. Quadrados médios da composição mineral de plantas de goiabeira submetidas a 30 e 50 dias de estresse salino.

\begin{tabular}{|c|c|c|c|c|}
\hline Variável & Tratamento & Período & Interação & Erro \\
\hline & \multicolumn{4}{|c|}{ Raiz } \\
\hline Sódio & $0,338 * *$ & $0,255^{* *}$ & $0,302 * *$ & 0,006 \\
\hline Cloro & $3,824 * *$ & $1,815^{* *}$ & $0,021^{\mathrm{ns}}$ & 0,014 \\
\hline Potássio & $0,049 * *$ & $0,381 * *$ & $0,016^{\mathrm{ns}}$ & 0,007 \\
\hline Cálcio & $0,048^{\mathrm{ns}}$ & $2,090 * *$ & $0,014^{\mathrm{ns}}$ & 0,031 \\
\hline \multirow[t]{2}{*}{ Magnésio } & $<0,001^{\mathrm{ns}}$ & $<0,001^{\mathrm{ns}}$ & $<0,001^{\mathrm{ns}}$ & $<0,001$ \\
\hline & \multicolumn{4}{|c|}{ Caule } \\
\hline Sódio & $0,049 * *$ & $0,041^{* *}$ & $0,008^{* *}$ & 0,010 \\
\hline Cloro & $0,779 *$ & $0,555^{* *}$ & $0,009^{\mathrm{ns}}$ & 0,007 \\
\hline Potássio & $0,021 * *$ & $0,542 * *$ & $0,019^{\text {ns }}$ & 0,004 \\
\hline Cálcio & $0,123^{*}$ & $<0,001^{\mathrm{ns}}$ & $0,008^{\mathrm{ns}}$ & 0,027 \\
\hline \multirow[t]{2}{*}{ Magnésio } & $0,001^{\mathrm{ns}}$ & $<0,001^{\mathrm{ns}}$ & $<0,0010^{\mathrm{ns}}$ & $<0,001$ \\
\hline & \multicolumn{4}{|c|}{ Folha } \\
\hline Sódio & $0,338 * *$ & $0,491 * *$ & $0,040 \mathrm{~ns}$ & 0,015 \\
\hline Cloro & $7,178^{* *}$ & $3,555^{* *}$ & $0,109^{*}$ & 0,016 \\
\hline Potássio & $0,688 * *$ & $0,005 * *$ & $0,003^{\mathrm{ns}}$ & 0,005 \\
\hline Cálcio & $0,359 * *$ & $0,555^{*}$ & $0,036^{\mathrm{ns}}$ & 0,078 \\
\hline Magnésio & $0,007 *$ & $0,003^{\mathrm{ns}}$ & $0,000^{\mathrm{ns}}$ & $<0,001$ \\
\hline
\end{tabular}

ns Não-significativo. ${ }^{*}$ Significativo a $5 \%$ de probabilidade pelo teste $\mathrm{F}$. ${ }^{* *}$ Significativo a $1 \%$ de probabilidade pelo teste $\mathrm{F}$.

Foi igualmente observado aumento significativo nos teores deste elemento entre as duas colheitas. $\mathrm{O}$ ajuste feito entre níveis de salinidade e teor de $\mathrm{Na}$ revelou uma relação linear, sendo os aumentos bem mais expressivos nas raízes e folhas, nas duas datas amostradas, ao passo que no caule o incremento foi mais reduzido (Figura 2). No tratamento-controle, os teores de $\mathrm{Na}$ foram muito próximos nos três órgãos, e com o aumento da salinidade notou-se uma divergência nas retas, com acréscimos expressivos muito próximos em relação a raiz e folha, e bem menores com relação ao caule, nos dois tempos de estresse salino.

Aumentos expressivos na concentração de Na nas raízes de goiabeira $(0,41$ para $0,70 \%)$ também foram encontrados por Patil et al. (1984) entre o controle e o nível de $60 \mathrm{mM}$ de $\mathrm{NaCl}$. Em espécies do gênero Annona foram observados aumentos superiores a $170 \%$ nos teores de $\mathrm{Na}$ nas folhas novas de plântulas submetidas a $60 \mathrm{mM}$ de $\mathrm{NaCl}$. 
(a)
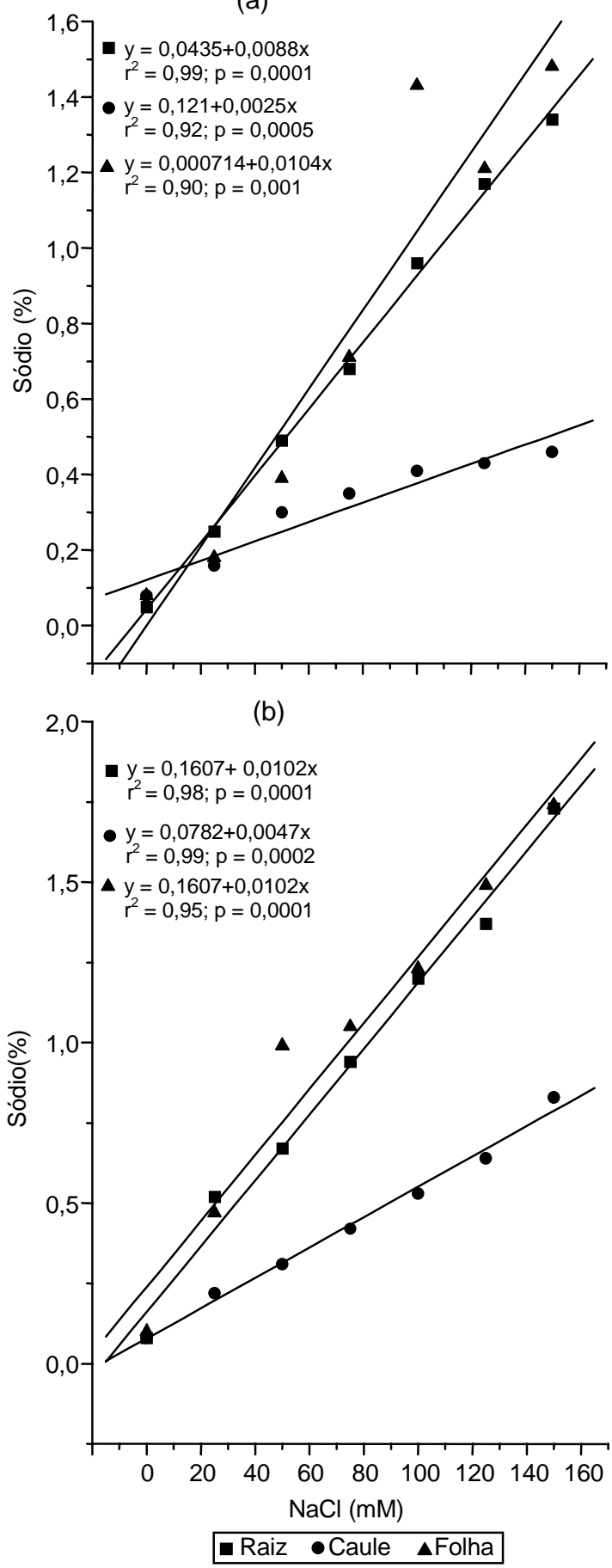

Figura 2. Porcentagem de sódio em raízes, caule e folhas de goiabeira em razão de níveis de $\mathrm{NaCl}$ na solução nutritiva após (a) 30 e (b) 50 dias de aplicação do estresse salino.
As concentrações encontradas foram menores em A. muricata do que em A. cherimola, esta última considerada tolerante (Ebert, 1998).

Os teores de $\mathrm{Cl}^{-}$nos tecidos aumentaram de forma significativa com os níveis de $\mathrm{NaCl}$ nas duas épocas de colheita (Tabela 1). Foi constatada uma relação linear direta entre níveis de salinidade e o teor deste elemento (Figura 3). Entre os órgãos estudados, as folhas apresentaram os maiores teores deste elemento, e o caule, os menores teores, enquanto as raízes apresentaram posição intermediária. Resultados semelhantes foram encontrados em espécies cítricas por Karstens et al. (1993) e Storey (1995). Ebert (1998) encontrou, em espécies de Annona submetidas a $60 \mathrm{mM}$ de $\mathrm{NaCl}$, resultados semelhantes. $\mathrm{O}$ autor comenta que há registros de mecanismos de retranslocação de $\mathrm{Cl}^{-}$de folhas jovens de macieira via xilema, de modo a evitar efeitos tóxicos à planta. Não está esclarecido se as lesões observadas nas folhas de plantas submetidas a estresse salino são devidas ao íon $\mathrm{Na}^{+}$ou $\mathrm{Cl}^{-}$. Em algumas espécies cítricas, o metabolismo é afetado pela presença de $\mathrm{Na}^{+}$ (Lloyd et al., 1990), enquanto em outras ele é afetado pelo $\mathrm{Cl}^{-}$(Bañuls \& Primo-Millo, 1992).

Os resultados obtidos sugerem não ter havido mecanismos de exclusão dos íons tóxicos $\left(\mathrm{Na}^{+} \mathrm{e} \mathrm{Cl}-\right)$ após o processo de absorção, resultando em acúmulo na parte aérea e clorose e queima nas folhas. Essas respostas resultaram, provavelmente, de alteração no balanço hormonal, na perda de turgescência das células-guarda e na redução generalizada da atividade metabólica da planta (Gorham et al., 1988).

Os teores de $\mathrm{K}$ foram reduzidos significativamente em todos os órgãos da planta com o aumento dos níveis de $\mathrm{NaCl}$ (Tabela 1). A Figura 4 mostra uma relação linear inversa entre os teores de $\mathrm{K}$ nos tecidos e níveis de $\mathrm{NaCl}$. As reduções nos teores deste elemento, em resposta à salinidade, foram mais acentuadas nas folhas que nos demais órgãos analisados.

Nas raízes e folhas, houve aumento significativo dos teores de $\mathrm{Ca}$, independentemente do nível de salinidade, entre as duas épocas de colheita (Tabela 1). Os níveis de $\mathrm{NaCl}$ determinaram reduções significativas nos teores de $\mathrm{Ca}$ apenas nas folhas e caule (Tabela 1). O decréscimo constatado foi linear, com o aumento da salinidade nas duas épocas de colheita (Figura 5). Para Cramer et al. (1987), o 

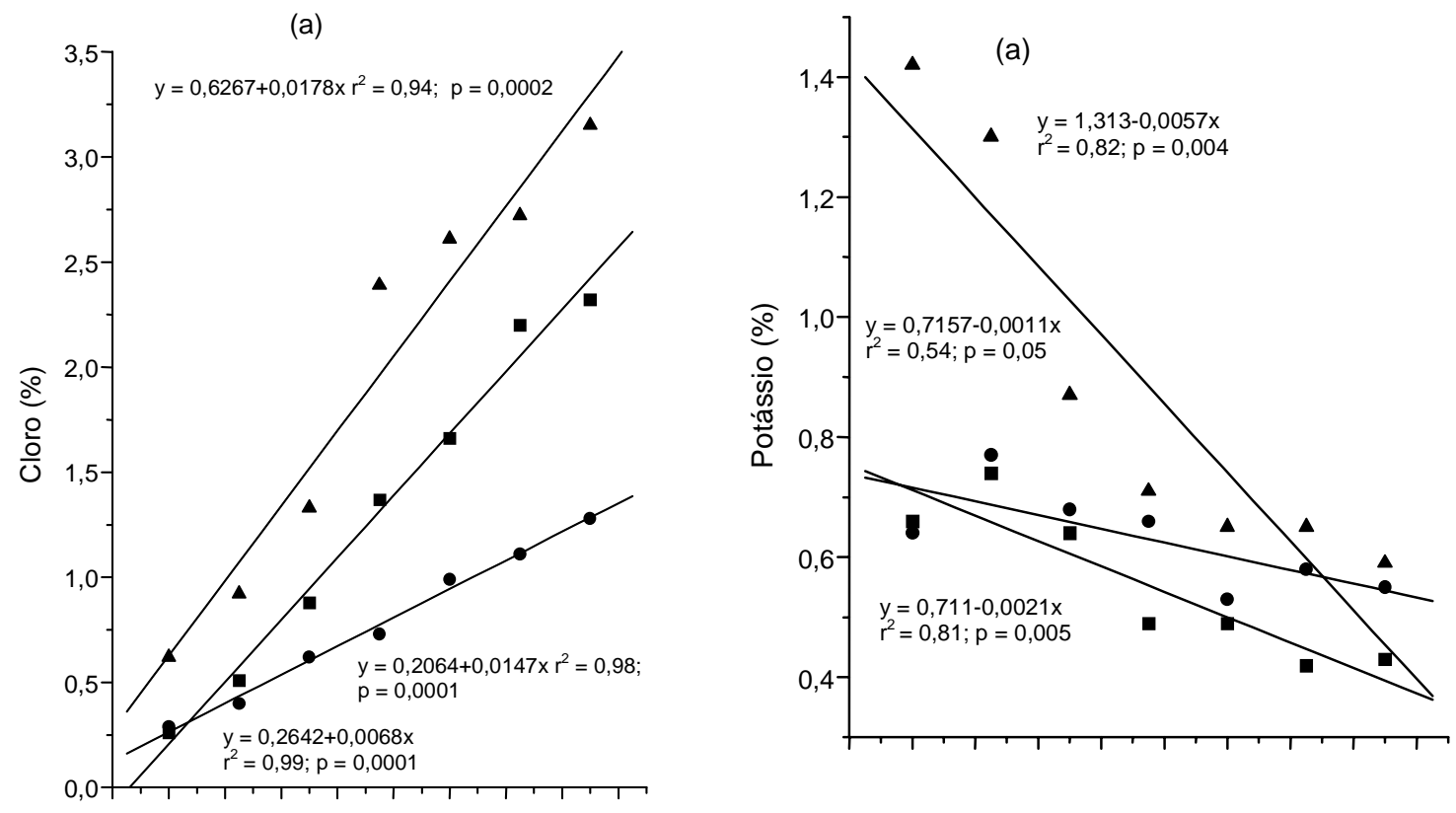

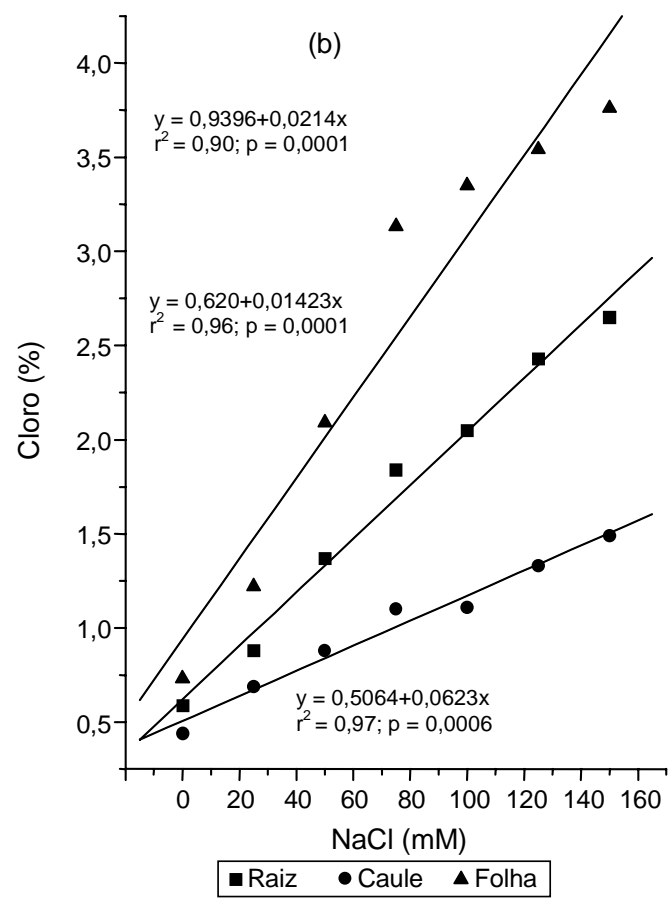

Figura 3. Porcentagem de cloro em raízes, caule e folhas de goiabeira em razão de níveis de $\mathrm{NaCl}$ na solução nutritiva após (a) 30 e (b) 50 dias de aplicação do estresse salino.

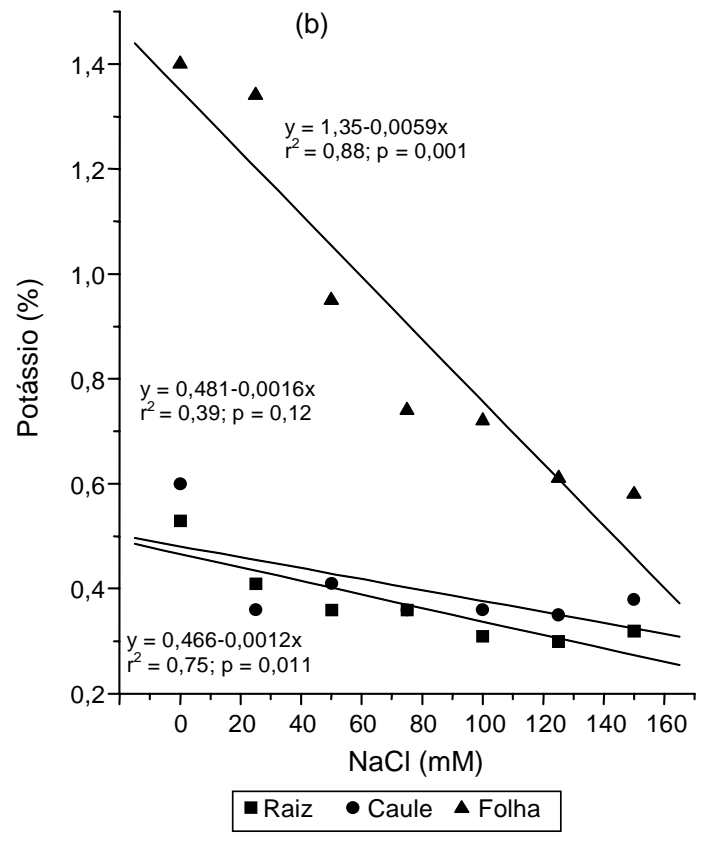

Figura 4. Porcentagem de potássio em raízes, caule e folhas de goiabeira em razão de níveis de $\mathrm{NaCl}$ na solução nutritiva após (a) 30 e (b) 50 dias de aplicação do estresse salino. 

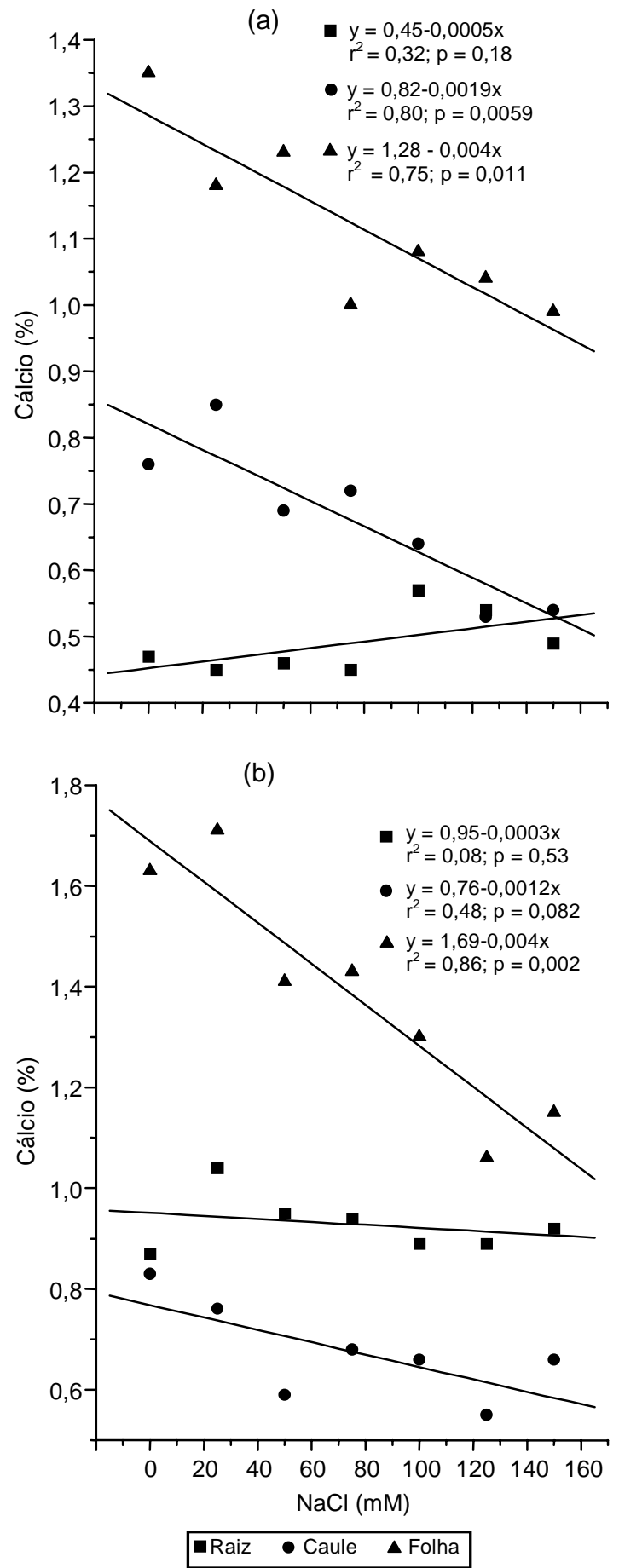

Figura 5. Porcentagem de cálcio em raízes, caule e folhas de goiabeira em razão de níveis de $\mathrm{NaCl}$ na solução nutritiva após (a) 30 e (b) 50 dias de aplicação do estresse salino.
Ca está envolvido na manutenção da integridade da membrana celular e desempenha importante papel na seletividade de absorção da relação $\mathrm{K} / \mathrm{Na}$.

Dos elementos estudados, o $\mathrm{Mg}$ foi o menos afetado pela salinidade. Não houve efeitos significativos da salinidade nos teores deste elemento nas raízes e no caule (Tabela 1). Na folhas, observou-se um decréscimo linear nos teores deste elemento com o aumento da salinidade (Figura 6). Fica evidente que os teores deste elemento nas folhas são superiores aos observados nos demais órgãos estudados, independentemente do nível de salinidade.

As alterações constatadas nos teores dos elementos estudados em determinados órgãos da planta refletem os efeitos prejudiciais do sal em relação à capacidade da planta em absorver e utilizar os nutrientes essenciais ao seu desenvolvimento (Leopold \& Willing, 1984). Shybank \& Kasshirad (1978), pesquisando os efeitos do $\mathrm{NaCl}$ sobre plantas de Acacia saligna, cultivada em diferentes concentrações (96 a $144 \mathrm{mM}$ ), observaram que os teores de $\mathrm{Na}$ e $\mathrm{Cl}$ no caule aumentaram com os níveis de $\mathrm{NaCl}$, e que as concentrações de $\mathrm{K}, \mathrm{Ca}$ e $\mathrm{Mg}$ tenderam a declinar com o aumento da salinidade. Há, entretanto, resultados divergentes, que mostram aumento nos teores de $\mathrm{Ca}$ e $\mathrm{K}$ na parte aérea. Esta aparente discrepância nos resultados é devida, provavelmente, a diferenças com relação à concentração de sal utilizada, à duração da imposição do estresse, e à espécie estudada (Greenway \& Munns,1980; Jeschke \& Wolf, 1988). Os resultados mostram um desequilíbrio nutricional causado pelo sal ao longo do experimento. O decréscimo na absorção de $\mathrm{K}$, associado ao aumento na concentração de $\mathrm{Na}$ nos tecidos das folhas aqui constatado, foi igualmente registrado por diversos autores em diferentes espécies (Jindal et al., 1979; Desai \& Sing, 1980; Patil \& Bhambota, 1980; Patil et al, 1984; Ebert, 1998). As maiores alterações para mais ou para menos, observadas nos teores dos elementos estudados, em resposta à salinidade, ocorreram nas folhas.

A Figura 7 relaciona os teores de $\mathrm{Na}$ com os dos demais elementos constituintes da composição química das raízes e folhas. Os dados se ajustaram a uma função polinomial de segundo grau. $\mathrm{O}$ teor de $\mathrm{Mg}$ apresentou pequena variação nas raízes e folhas, independentemente dos teores de $\mathrm{Na}$. Os teores de $\mathrm{Cl}$ 
cresceram quase linearmente com os de Na nas raízes e folhas. $\mathrm{O}$ teor de $\mathrm{K}$ apresentou comportamento semelhante nas raízes e folhas, com uma leve tendência para queda com o aumento do teor de $\mathrm{Na}$. A relação entre os teores de $\mathrm{Ca}$ e $\mathrm{Na}$ não foi signi-

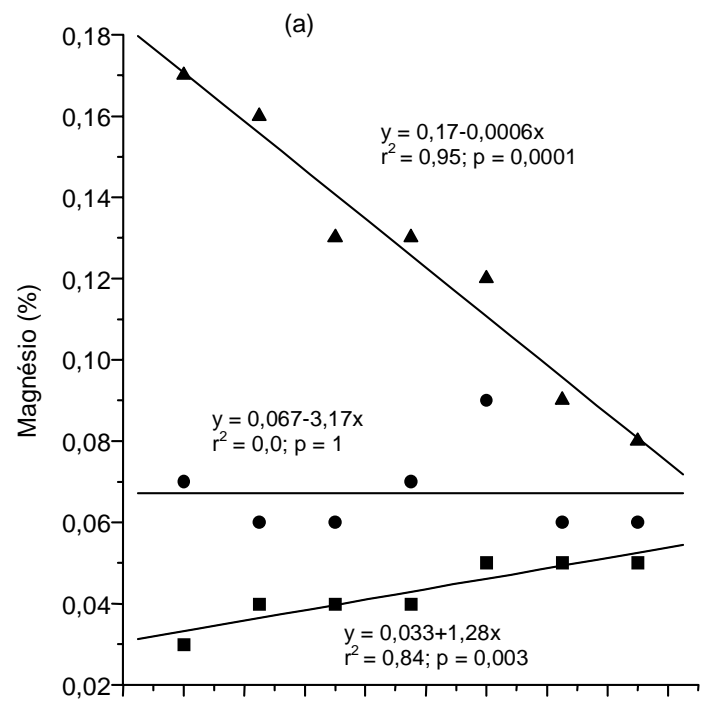

(b)

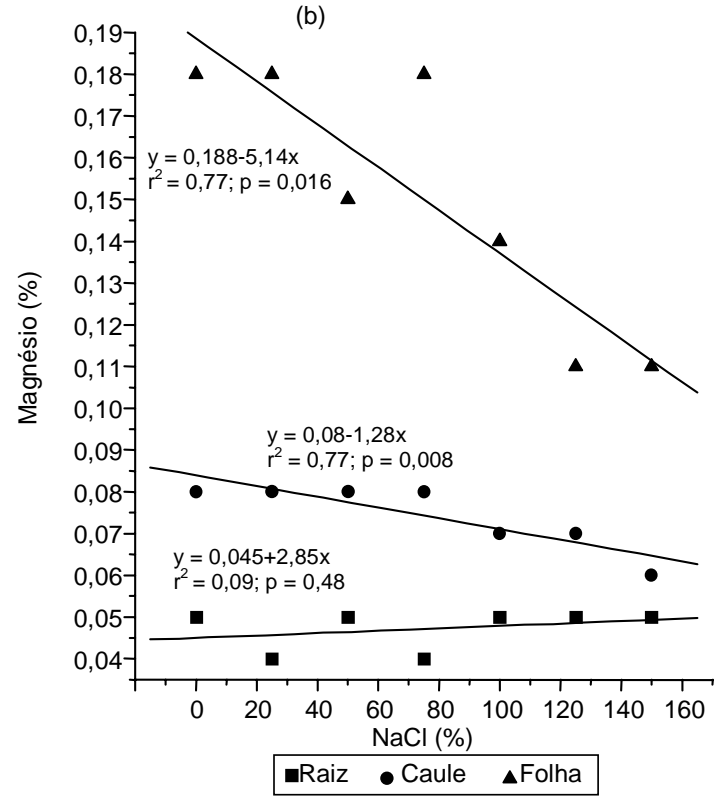

Figura 6. Porcentagem de magnésio em raízes, caule e folhas de goiabeira em razão de níveis de $\mathrm{NaCl}$ na solução nutritiva após (a) 30 e (b) 50 dias de aplicação do estresse salino. (a)

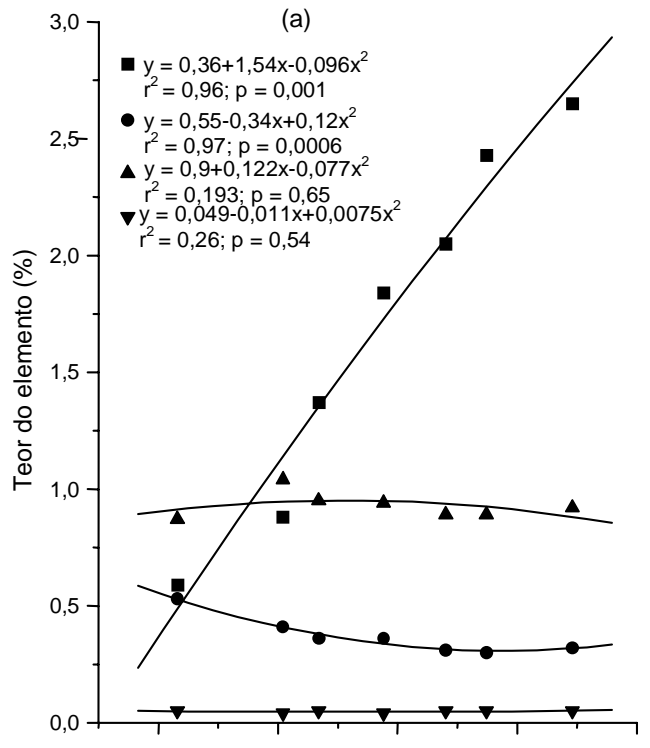

(b)

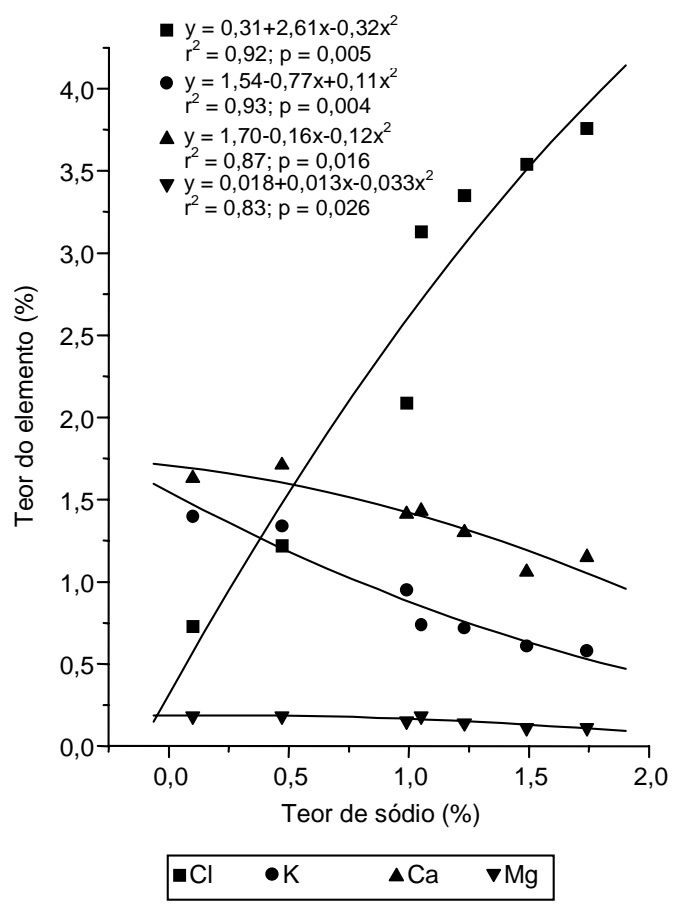

Figura 7. Relação entre os teores de $\mathrm{Na}$ e os teores de CI, K, Ca e Mg nas folhas (a) e raízes (b) de plantas de goiabeira submetidas a 50 dias de estresse com diferentes níveis de $\mathrm{NaCI}$. 
ficativa. Tais resultados comprovam, em parte, as interações comentadas por Bernstein (1975), que relatam que a deficiência de $\mathrm{Ca}^{2+}$ pode ser induzida pelo excesso de $\mathrm{Na}^{+}$e a de $\mathrm{K}^{+}$por excesso de $\mathrm{Na}^{+}$ou $\mathrm{Ca}^{+}$.

\section{Conclusões}

1. A redução na produção de matéria seca em goiabeira submetida a estresse salino com $\mathrm{NaCl}$ está associada ao aumento dos teores de $\mathrm{Na}$ e $\mathrm{Cl}$ nas folhas, raízes e caule da planta.

2. O estresse salino com $\mathrm{NaCl}$ causa um desequilíbrio nutricional, evidenciado por reduções nos teores de $\mathrm{K}, \mathrm{Ca}$ e $\mathrm{Mg}$ na matéria seca das raízes, caule e folhas de goiabeira.

3. A goiabeira mostra-se moderadamente tolerante ao estresse salino, apresentando reduções no crescimento a partir da concentração de $50 \mathrm{mM}$ de $\mathrm{NaCl}$.

\section{Referências}

BAÑULS, J.; PRIMO-MILLO, E. Effects of chloride and sodium on gas exchange parameters and water relations of citrus plants. Physiologia Plantarum, Copenhagen, v. 86, p. 115-123, 1992.

BERNSTEIN, L. Effects of salinity and sodicity on plant growth. Annual Review of Phytopathology, Palo Alto, v. 13, p. 295-312, 1975.

CRAMER, G. R.; LAUCHLI, A.; EPSTEIN, E. Influx of $\mathrm{Na}^{+}, \mathrm{K}^{+} \mathrm{e} \mathrm{Ca}^{2+}$ into roots of salt-stressed cotton seedlings. Physiologia Plantarum, Copenhagen, v. 83, p. 510-516, 1987.

DESAI, U. T.; SINGH, R. M. Growth of guava plants (Psidium guajava L.) as affected by salinity. Indian Journal of Horticulture, Bangalore, v. 5, p. 3-6, 1980.

DEVITT, D. A.; MORRIS, R. L. Morphological response of flowering annuals to salinity. American Society for Horticulture Science Journal, Alexandria, v. 112, p. 951955, 1987.

EBERT, G. Growth, ion uptake and gas exchange of two Annona species under salt stress. Angewandte Botanik, Berlin, v. 72, p. 61-65, 1998.
FLOWER, T. J.; YEO, A. R. Ion relations of plants under drought and salinity. Australian Journal of Plant Physiology, Collingwood, v. 113, p. 75-79, 1986.

GORHAM, J.; TOMAR, O. S.; JONES, G. R. Salinity induced changes in the chemical composition of Leucaena leucocephala and Sesbania bispinosa. Plant Physiology, Rockville, v. 132, p. 678-682, 1988.

GREENWAY, H.; MUNNS, R. Mechanism of tolerance in non-halophytes. Annual Review of Plant Physiology, Palo Alto, v. 31, p. 49-190, 1980.

HOAGLAND, D. R.; ARNON, D. I. The water culture method for growing plants without soil. Berkeley : University of California, 1950. 31 p. (Circular, 347).

HOFFMAN, G. J.; RAWLINS, S. L.; GARBER, M. J.; CULEN, E. M. Water relations and growth of cotton as influenced by salinity and relative humidity. Agronomy Journal, Madison, v. 63, p. 822-826, 1971.

JARREL, W. M.; VIGINIA, R. A. Response of mesquite to nitrate and salinity in a simulated phreatic environment: water use, dry mater and mineral nutrient accumulation. Plant and Soil, Dordrecht, v. 125, p. 185-196, 1990.

JESCHKE, W.; WOLF, D. Effect of $\mathrm{NaCl}$ salinity on growth, development, ion distribution, and ion translocation in caston bean (Ricinus communis L.). Plant Physiology, Rockville, v. 132, p. 45-53, 1988.

JINDAL, P. C.; SINGH, J. P.; GUPTA, O. P. Effect of salinity on the mineral nutrients in mango seedlings. Indian Journal of Agriculture Sciences, New Delhi, v. 49, p. 105-109, 1979.

KARSTENS, G. S.; EBERT, G.; LUDDERS, P. Long-term and short-term effects of salinity on root respiration, photosynthesis and transpiration of citrus rootstocks. Angewandte Botanik, Berlin, v. 67, p. 3-8, 1993.

LEOPOLD, A. C.; WILLING, R. D. Evidence of toxicity and effects of salt on membranes. In: STAPLES, R. C.; TOENNIESSEN, G. H. (Ed.). Saline tolerance in plants. New York : J. Willey, 1984. 820 p.

LLOYD, A.; KRIEDEMANN, P. E.; ASPINALL, A. Contrast between citrus species in response to salinization: an analysis of photosynthesis and water relations for different rootstocks-scion combination. Physiologia Plantarum, Copenhagen, v. 78, p. 236-246, 1990.

MALAVOLTA, E.; VITTI, C.; OLIVEIRA. S. de. Avaliação do estado nutricional das plantas: princípios e aplicações. Piracicaba : Potafos, 1989. 201 p. 
MIYAZAWA, M.; PAVAN, M. A.; BLOCH, M. de F. M. Avaliação de métodos com e sem digestão para extração de elementos em tecidos de plantas. Ciência e Cultura, São Paulo, v. 36, n. 11, p. 1953-1958, 1984.

PATIL, V. K.; BHAMBOTA, J. R. Salinity studies in citrus. I. Effect of various levels of salinity on the micronutrients status of seedlings root stock of citrus. Indian Society of Soil Science Journal, New Delhi, v. 28, p. 72-79, 1980.

PATIL, P. K.; PATIL, Y. K.; GHONSIKAR, C. P. Effect of soil salinity on growth and nutritional status of guava (Psidium guajava L.). International Journal Tropical Agriculture, Hissar, v. 2, n. 4, p. 337-344, 1984.

RHOADES, J. D.; LOVEDAY, J. Salinity in irrigated agriculture. In: STEWART, B. A.; NIELSEN, D. R. (Ed.). Irrigation of agricultural crops. Madison : American Society of Agronomy, 1990. p. 1089-1157. (ASA. Monograph, 30).

SHANNON, M. C.; RHOADES, J. H.; DRAPER, J. H.; SCARDACI, S. C.; SPYRES, M. D. Assessment of salt tolerance in rice cultivars in response to salinity problems in California. Crop Science, Madison, v. 38, p. 394-398, 1998.
SHYBANK, L. C.; KASSHIRAD, M. R. Salinity and citrus yield: an analysis of results from a salinity survey. Journal of Horticultural Science, Ashford, v. 49, p. 1527, 1978.

STOLZY, D. L. L.; JARREL, W. M. Response of sorghum and wheat to different $\mathrm{K}^{+} / \mathrm{Na}^{+}$ratios at varying osmotic potentials. Agronomy Journal, Madison, v. 76, p. 681688, 1983.

STOREY, R. Salt tolerance, ion relations and the effect of root medium on the response of citrus to salinity. Plant Physiology, Rockville, v. 22, p. 1-14, 1995.

TSUCHIYA, M.; MIYAKE, M.; HITOSHI, N. Physiological response to salinity in rice plant. III. A possible mechanism for $\mathrm{Na}^{+}$exclusion in rice root under $\mathrm{NaCl}$-stress conditions. Japanese Journal of Crop Science, Tokyo, v. 63, p. 326-332, 1994.

YANG, Y. W.; NEWTON, J. R.; MILLER, F. R. Salinity tolerance in sorghum whole plant response. Crop Science, Madison, v. 30, p. 775-781, 1990.

YEO, R. A.; FLOWER, T. J. Salt tolerance in the halophyte Suaeda maritima L. evaluation of the effect of salinity grow. Journal of Experimental Botany, Oxford, v. 31, p. 117-183, 1989. 